\title{
Conduction Mechanism of Charge Carriers in Electrodes and Design Factors for the Improvement of Charge Conduction in Li-ion Batteries
}

\author{
Sophia Akhtar ${ }^{1}$, Wontae Lee ${ }^{1,3}$, Minji Kim ${ }^{1}$, Min-Sik Park ${ }^{2 *}$, and Won-Sub Yoon ${ }^{1 *}$ \\ ${ }^{1}$ Department of Energy Science, Sungkyunkwan University, Suwon 440-746, Korea \\ ${ }^{2}$ Department of Advanced Materials Engineering for Information and Electronics, Kyung Hee University, Yongin, 17104, Korea \\ ${ }^{3}$ The Institute of New Paradigm of Energy Science Convergence, Sungkyunkwan University, Suwon 16419, Korea
}

\begin{abstract}
In-depth knowledge of electrode processes is crucial for determining the electrochemical performance of lithium-ion batteries (LIBs). In particular, the conduction mechanisms of charged species in the electrodes, such as lithium ions $\left(\mathrm{Li}^{+}\right)$and electrons, are directly correlated with the performance of the battery because the overall reaction is dependent on the charge transport behavior in the electrodes. Therefore, it is necessary to understand the different electrochemical processes occurring in electrodes in order to elucidate the charge conduction phenomenon. Thus, it is essential to conduct fundamental studies on electrochemical processes to resolve the technical challenges and issues arising during the ionic and electronic conduction. Furthermore, it is also necessary to understand the transport of charged species as well as the predominant factors affecting their transport in electrodes. Based on such in-depth studies, potential approaches can be introduced to enhance the mobility of charged entities, thereby achieving superior battery performances. A clear understanding of the conduction mechanism inside electrodes can help overcome challenges associated with the rapid movement of charged species and provide a practical guideline for the development of advanced materials suitable for high-performance LIBs.
\end{abstract}

Keywords : Li-ion Battery, Charge Transport Mechanism, Ionic Conduction, Electron Conduction

Received : 16 October 2020, Accepted : 28 November 2020

\section{Introduction}

Lithium (Li)-ion batteries (LIBs) are promising sources of power for various applications ranging from electronic devices to plug-in hybrid electric vehicles [1-4]. For the successful implementation of LIBs, it is crucial to meet the rigorous requirements of the industry. As far as the operation is concerned, LIBs should be safe and there must not be a fire hazard. [5]. A typical LIB is primarily composed of porous electrodes [6-7] (i.e., active material, conductive additive, and binder coated on the current collectors), an electrolyte, and a separator to prevent a short-circuit during operation. Multiple processes occur inside these

*E-mail address: wsyoon@skku.edu, mspark@khu.ac.kr DOI: https://doi.org/10.33961/jecst.2020.01564

This is an open-access article distributed under the terms of the Creative Commons Attribution Non-Commercial License (http://creativecommons.org/licenses/by-nc/4.0) which permits unrestricted non-commercial use, distribution, and reproduction in any medium, provided the original work is properly cited. porous electrodes during the operation of LIBs [8].

Generally, there are two types of charge transfer processes occurring inside electrodes that govern the appropriate operation of LIB; the first process is the electron transfer and the second process is the ion transfer. However, it is necessary to understand the percolation path of these charged entities at the interfaces when they move simultaneously during the operation of the battery.

The electrons are supplied by the current collectors for electrodes, while lithium ions $\left(\mathrm{Li}^{+}\right)$move through the active material and pores, thereby creating a pathway through the electrolyte. It should be noted that electron transfer through the electrolyte is undesirable because it could cause severe damage to the cell and may result in the explosion of the cell, leading to serious hazards. The electronic conduction in electrodes should occur fast; therefore, a conducting additive is generally used alongside the active materials. The suitable current collectors that 


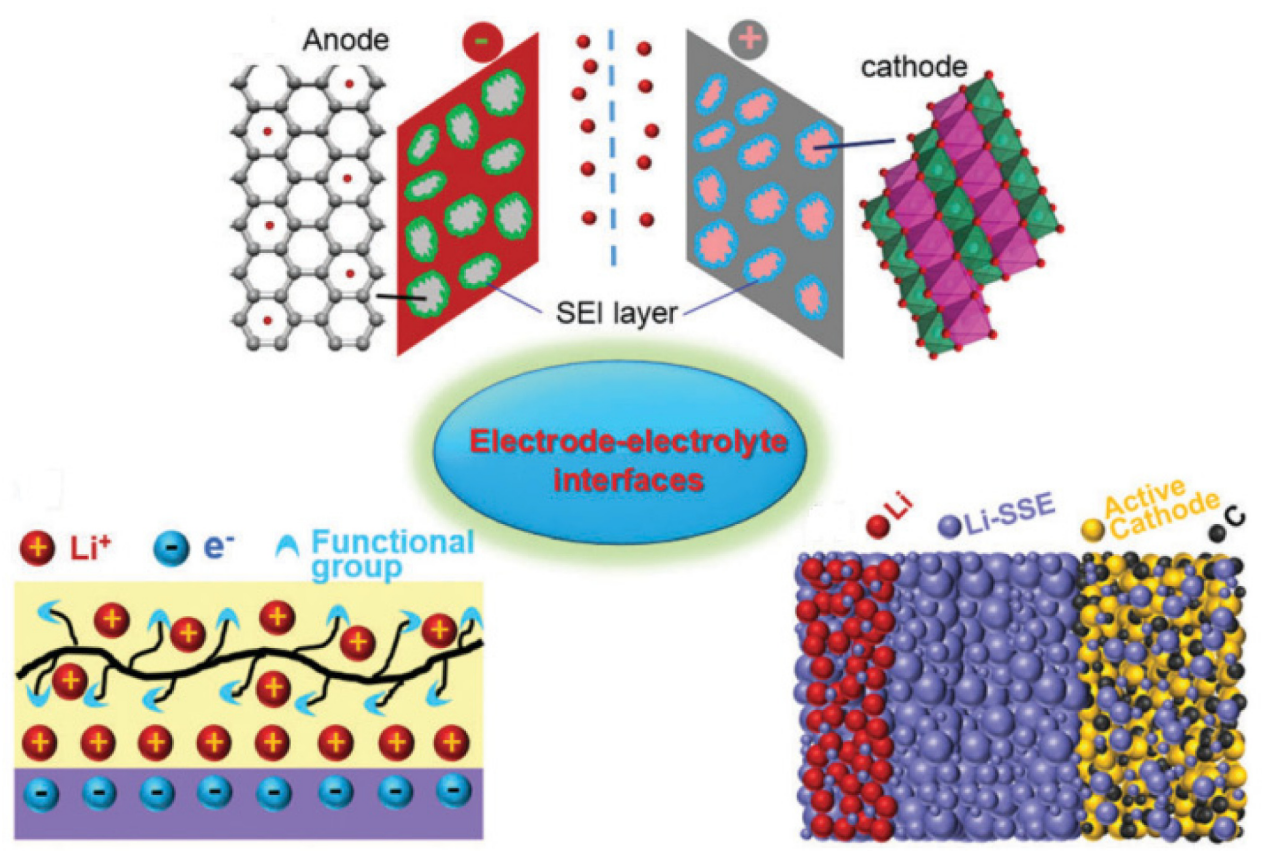

Fig. 1. Electrode-electrolyte interfaces in Li-ion, Li-polymer, and all solid-state Li-based batteries. The figures were reproduced with permission from Ref. [10]. Copyright 2018, Energy and Environmental Science.

can lead to rapid electron movement are aluminum (Al) foil for the cathode and copper $(\mathrm{Cu})$ for the anode.

Further understanding of the charge transfer reactions at the electrode-electrolyte interface is crucial to control the reaction kinetics [9]. The reactions that do not occur at the electrode-electrolyte interface are typically irreversible and affect the performance of the electrochemical cell [10]. Hence, the overall reaction must only occur at the interface.

The interfacial reactions are mainly responsible for determining the reversibility of the electrochemical reactions in LIBs. The reversibility of electrochemical reactions directly reflects on the coulombic efficiency, although it does not significantly affect the energy density of LIBs [10-11].

Charge transport mechanisms in the electrodes are highly dependent on the properties of active materials, because electrochemical redox reactions mainly occur at the surface of these materials with the electron transport coupled to $\mathrm{Li}^{+}$transport. Therefore, the rate-limiting factor could either be ion or electron transport at the electrodes, depending on the active materials [12].
Both ionic and electronic conduction at the electrodes are rate-limiting steps because the internal resistance of the electrodes affects the conduction path of both $\mathrm{Li}^{+}$and electrons. The electronic transport is affected by the film resistance of current collectors, conducting additives, and surface resistance induced by the active materials in the electrodes. In contrast, ionic transport is mainly governed by the solid-state diffusion of $\mathrm{Li}^{+}$in the active materials as well as the ionic resistance of the electrolyte. Consequently, both $\mathrm{Li}^{+}$and electron conduction significantly affect the overall performance of LIBs [5].

$\mathrm{Li}^{+}$and electron transport follow a 3D complex tortuous path in the electrodes. A complex tortuous path for the charged species is generally formed in electrodes impregnated with the electrolyte and consisting of current collectors coated with an active material, a conductive additive, and a polymeric binder. Generally, electron transport occurs through the conductive additive and active material, whereas $\mathrm{Li}^{+}$transport occurs through the electrolyte-filled pores. Thus, $\mathrm{Li}^{+}$and electron transport may follow a longer path when compared with the thickness of an electrode [13-16]. 


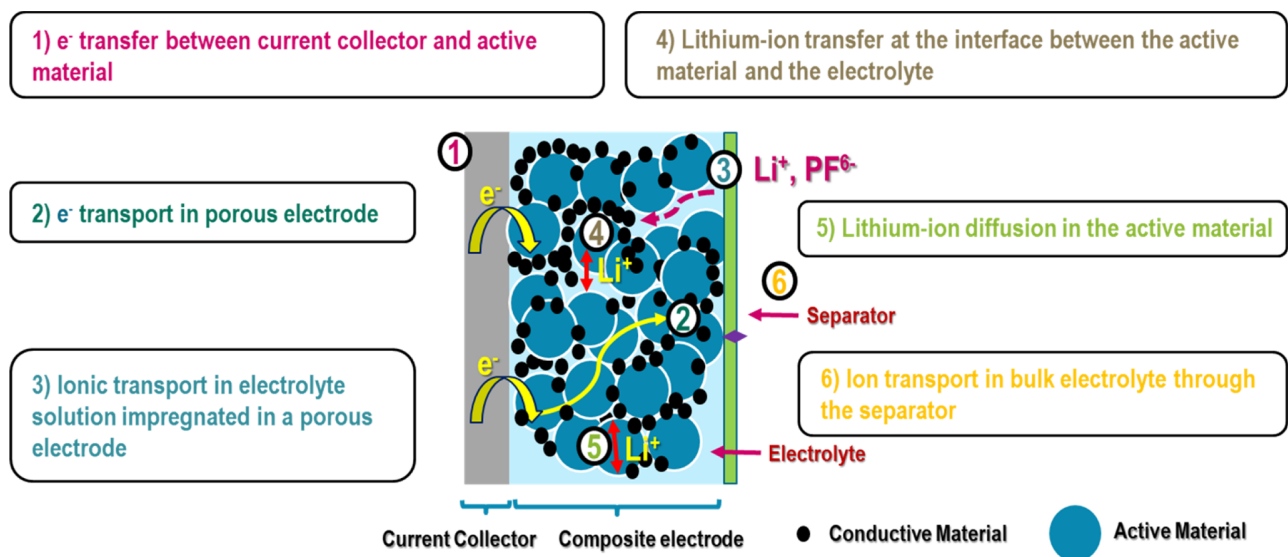

Fig. 2. Electrochemical processes occurring within a Li-ion battery electrode.

Electrode processes typically govern the working of a cell. The internal resistance of every part of the electrode restricts the movement of $\mathrm{Li}^{+}$and electrons. This resistance is offered by the electrode [8] during (1) electron transfer at the boundary of the active material and current collector, (2) electron transport in the porous electrode, (3) ion transport in the electrolyte solution infused in the porous electrode, (4) $\mathrm{Li}^{+}$transfer at the active material/electrolyte interface, (5) $\mathrm{Li}^{+}$diffusion in the active material, and (6) ion transport in the electrolyte through the separator. Electronic conduction and ionic diffusion related electrochemical processes are explained in chapter 2 and 3 of this review, respectively.

\section{Electron Conduction and Transport Mech- anism}

According to the band theory, the electronic conduction behavior of materials can be interpreted by analyzing their electronic band structures, as illustrated by quantum mechanics. In general, the materials have distinct band structures, which are predominant features for determining the electrical properties of materials such as insulators, conductors, and semiconductors. Conductors have partially filled valence bands, whereas semiconductors have overlapped bands, thereby enabling electrons to move in a crystal upon the application of an external field. Moreover, metals exhibit excellent electrical conductivity because they have zero band gap.

In LIBs, metals that exhibit excellent electrical conductivity, such as $\mathrm{Cu}$ and $\mathrm{Al}$, are generally employed as the current collectors. Active materials (i.e., insulators) usually possess a large band gap (e.g., lithium cobalt oxide $\left(\mathrm{LiCoO}_{2}\right)$ has a bandgap of $0.5-2.7 \mathrm{eV}$ and electrical conductivity of $\sim 10^{-4}$ ); hence, electron movement does not occur due to the filled valence and empty conduction bands [12]. The electronic conduction characteristics of active materials can be described using two approaches: classical transport theory and quantum transport theory.

\subsection{Classical approach}

The classical transport theory considers electrical conduction as a flow of free electrons. Under the application of an external electric field and the internal frictional force, the flow of free electrons attains equilibrium, and a constant current density is observed. By implementing the time relaxation approximation, the electrical conductivity can be expressed as follows [17]:

$$
\sigma=\frac{N_{f} e^{2} \tau}{m}
$$

where $\sigma$ is the electrical conductivity, $\tau$ is the relaxation time, $\mathrm{N}_{\mathrm{f}}$ is the number of free electrons, $\mathrm{m}$ is the mass of an electron, and e is the charge of an electron. A classical approximation is generally used to describe the typical electrical conduction in metals [18].

\subsection{Quantum mechanical approach}

The quantum mechanical approach states that only 
the electrons that are located near the Fermi level contribute to $\sigma$ of the materials. Such electrons near the Fermi level can be accelerated via an external electrical field. Thus, electron movement only occurs along the direction of the applied field. The displaced free electrons possess equal or slightly higher velocities than the Fermi velocity $(\gamma \mathrm{F})[12]$. These free electrons could participate in the electron conduction; hence, $\sigma$ for a quantum mechanical system can be expressed as

$$
\sigma=\frac{1}{3} e^{2} v_{F}^{2} \tau N\left(E_{F}\right)
$$

Equation (2) describes the dependence of $\sigma$ on the $\gamma \mathrm{F}$ of free electrons, $\tau$, and density of states. The abovementioned equation explains that $\sigma$ is directly dependent on $\tau$ and the average time between successive collisions of free electrons [18].

$\sigma$ can also be explained in terms of the absolute value of e and the number of conducting electrons, as expressed in equation (3) [17]. Electron mobility is a useful entity for describing the transport or conduction of electrons.

$$
\sigma=n|e| \mu_{e}
$$

Quantum mechanics provides a range of concepts for a clear understanding of the influence of dopants and impurities in materials on altering carrier concentrations. The quantum mechanical expression for conductivity considers the electrons and density of states near the Fermi level; hence, it is more advantageous as compared to the classical model.

\subsubsection{Electron tunneling and percolation path}

Electron tunneling is an exponential function of the distance as well as the inverse barrier height for electron transfer. The probability of electron tunneling tends to decrease exponentially with an increase in the height and width of the electron transfer barrier.

$$
T=\left|\frac{F}{A}\right|^{2} \approx \frac{16 E_{o}\left(V-E_{o}\right)}{V^{2}} e^{-2 \kappa L}
$$

In equation (4), $\mathrm{T}$ is the probability that an electron will tunnel through the barrier, $E_{o}$ is the total energy of an incoming electron, $\mathrm{V}$ is the height of the potential barrier, $\mathrm{L}$ is the width of the potential barrier, and $\kappa$ is the wave number.

An efficient electron transfer between the active material and the current collector affects the kinetic performance of the working electrode. In addition, electron tunneling and percolation throughout the active material also affects the electrochemical performance of the cell.

\subsection{Conduction in active materials}

2.3.1 Electron transfer between the current collector and active material

In general, an increase in $\mathrm{V}$ reduces the probability of electron tunneling during the operation of LIBs. Pan et al. demonstrated the presence of electronic conductive $\mathrm{Cu}_{2} \mathrm{O}$ and insulated $\mathrm{Al}_{2} \mathrm{O}_{3}$ surface oxidation layers on the surface of $\mathrm{Cu}$ and $\mathrm{Al}$ foils, respectively. The insulated $\mathrm{Al}_{2} \mathrm{O}_{3}$ layer forces the electrons to tunnel through the $\mathrm{Al}_{2} \mathrm{O}_{3}$ surface layer on the surface of $\mathrm{Al}$ foil. As a result, the working capacity of $\mathrm{Li}_{4} \mathrm{Ti}_{5} \mathrm{O}_{12}$ (LTO) on the $\mathrm{Al}$ foil was found to decrease due to the kinetic limitation at a high current density [19].

Yang et al. [20] examined the feasibility of a 3D$\mathrm{NiCrAl}$ alloy foam as a current collector for $\mathrm{LiFePO}_{4}$ cathode material. The metal foam current collector enables efficient redox reactions owing to the shorter migration path of electrons and the large active surface area in the electrode. It should be noted that this short migration path of electrons and large surface area were advantageous for reducing the charge overpotential during the cycles, because electrons do not need to travel towards the current collector, similar to conventional foil-type current collectors.

The 3D-NiCrAl alloy foam features a roughened surface, which improves its adhesion with the active material, thereby reducing the interfacial resistance between the current collector and the active material.

On comparing the charge/discharge curves in Fig. 3(a), it is evident that there is no major difference in the charge overpotential in the plateau region of the two types of current collectors, at a low current density. At a high current density (Fig. 3(b)), however, a significant increase in the charge overpotential is observed in the plateau region of a foil-type current collector.

In addition, a significant capacity loss is observed in the foil-type current collector at a high current density due to the limited $\mathrm{Li}^{+}$diffusion. In contrast, the metal foam current collector shows significantly lower capacity loss even at a high current density, as shown in Fig. 3(c). The increase in the internal resis- 
(a)

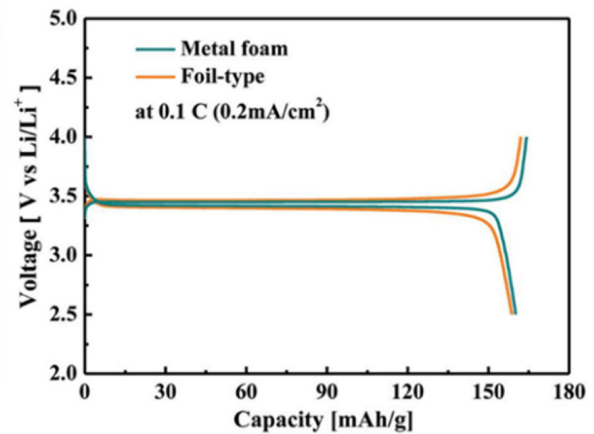

(c)

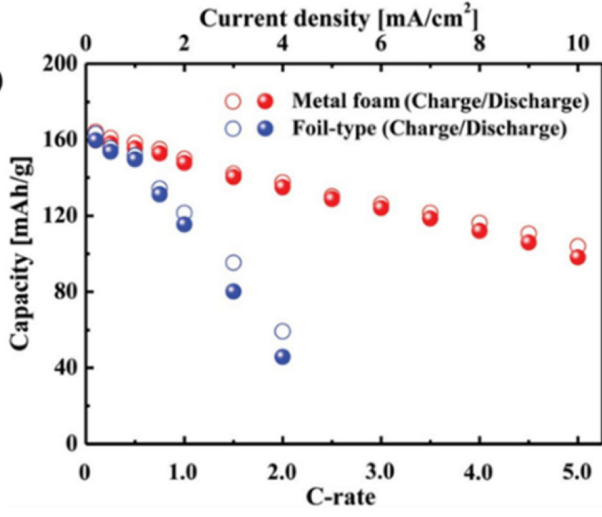

(b)
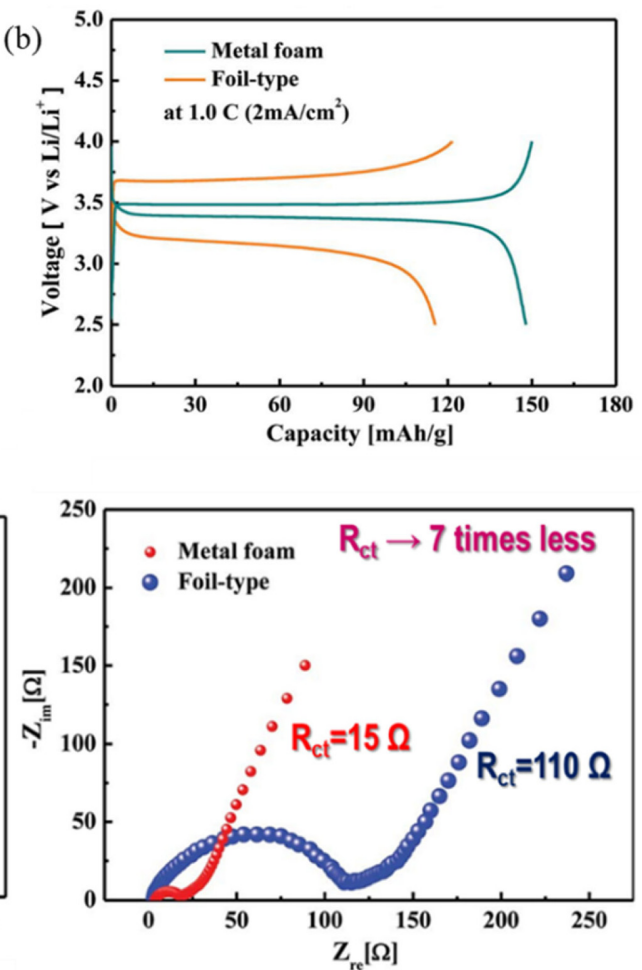

Fig. 3. Electrochemical performance of metal foam and foil-type current collector at (a) $0.1 \mathrm{C}$ and (b) $1.0 \mathrm{C}$. (c) Cyclic stability and electrochemical impedance spectroscopic curves for metal foam and foil-type current collectors. The figures were reproduced with permission from Ref. [20]. Copyright 2014, Journal of Material Chemistry A.

tance restricted the performance of the foil-type current collector.

The improved electrochemical performance is ascribed to the lower impedance and smaller polarization arising from the metal foam current collector. The metal foam exhibits a considerably smaller charge transfer resistance $\left(\mathrm{R}_{\mathrm{ct}}\right)$ at a high current density, as compared to the foil-type current collector [20].

\subsubsection{Electron transport in porous electrodes}

The high electronic conductivity of active materials is essential for the electron transfer in electrodes. Typically, additional carbon coatings are adopted for active materials with poor electronic conductivity, such as $\mathrm{LiFePO}_{4}$. The carbon coatings form electron percolation pathways in the electrode, resulting in improved electronic conductivity (Fig. 4).

A uniform carbon coating on the nanoporous micro- $\mathrm{LiFePO}_{4}$ cathode material can effectively form electron percolation pathways in the electrode, lead- ing to a substantial increase in electronic conductivity. As a result, the volumetric capacity of nanoporous micro- $\mathrm{LiFePO}_{4}$ is 2.5 times higher than that of the nano-LiFePO 4 (Fig. 5(a-c)). As shown in Fig. 5(d-e), nanoporous micro- $\mathrm{LiFePO}_{4}$ exhibits higher gravimetric and volumetric capacities at a high current rate.

The mutual effect of morphology and high electronic conductivity of nanoporous micro- $\mathrm{LiFePO}_{4}$ after carbon coating might be responsible for the higher gravimetric and volumetric capacities. The nanoporous particles combine the high capacity and high current rate of nano-sized particles with the high volumetric energy density of micro-sized particles [21].

\section{Ionic Diffusion and Transport Mechanism}

3.1 Macroscopic and microscopic diffusion processes Generally, ionic transport occurs under the influ- 
Highly crystalline carbon-coated nanoporous micro LiFePO

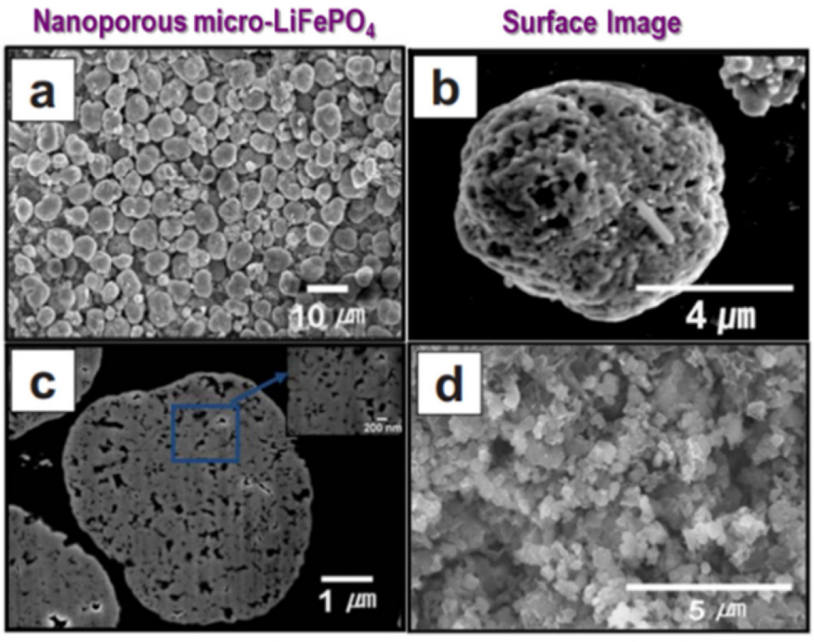

Cross-sectional Image

Nano-LiFePO 4 (e)

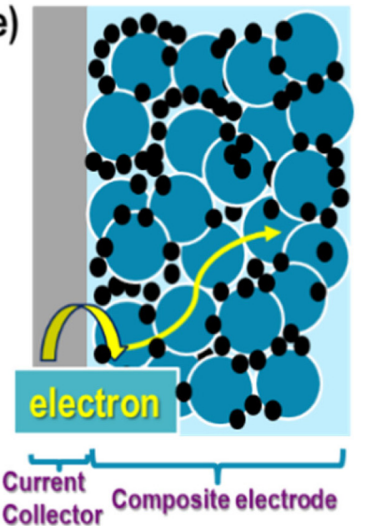

- Conductive Material Active Material

Fig. 4. (a-d) SEM micrographs of nanoporous micro- $\mathrm{LiFePO}_{4}$ and nano- $\mathrm{LiFePO}_{4}$, and (e) schematic diagram of electronic conduction through the active material. The figures were reproduced with permission from Ref. [21]. Copyright 2009, Electrochemical Solid-State Letters.

(a)

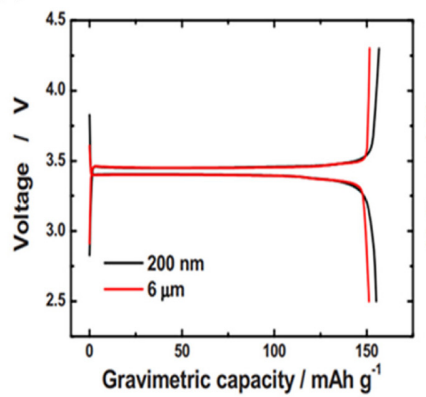

(d)

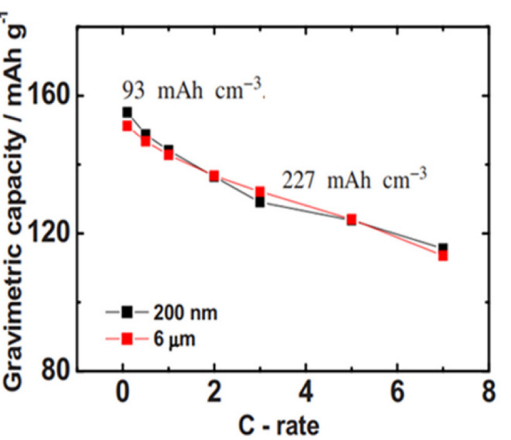

(b)

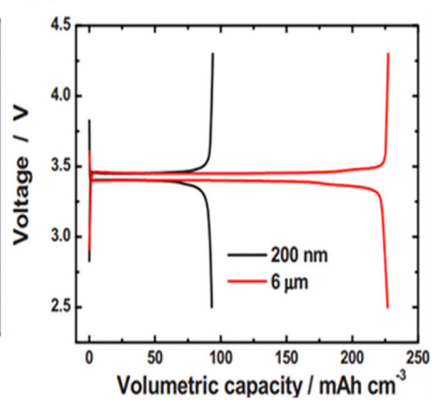

(c)

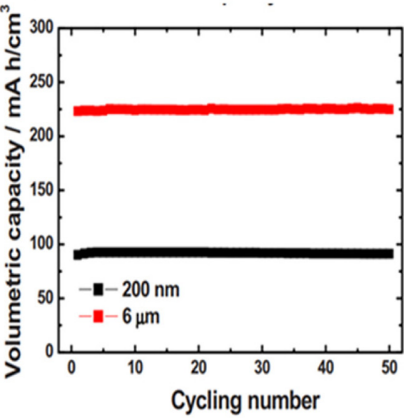

(e)

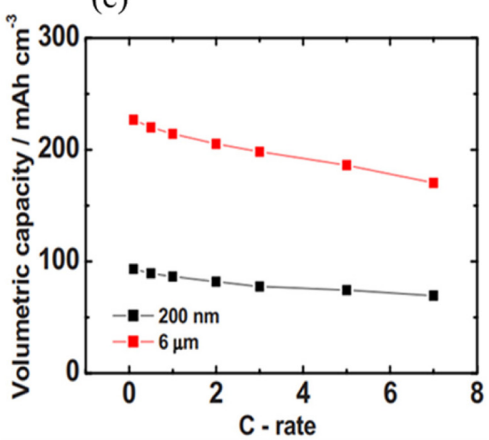

Fig. 5. Charge-discharge curves for (a) gravimetric capacity and (b) volumetric capacity, and (c) cyclability of nano$\mathrm{LiFePO}_{4}$ and nanoporous micro-LiFePO . (d) Gravimetric and (e) volumetric capacities of nano- $\mathrm{LiFePO}_{4}$ and nanoporous micro-LiFePO . The figures were reproduced with permission from Ref. [21]. Copyright 2009, Electrochemical Solid-State Letters. 
ence of different gradients such as the electrical field, chemical potential, and concentration. On a macroscopic scale, diffusion can be better explained by Fick's first law, as expressed by equation 5 [22]:

$$
J=-D V C
$$

where $\mathrm{J}$ is the flux of particles, $\mathrm{C}$ is the particle concentration, and $\mathrm{D}$ is the chemical diffusion coefficient. The negative sign specifies the gradient of the diffusion process, typically from higher to lower concentrations.

At the microscopic scale, the self-diffusion coefficient is more useful because it provides additional information regarding the intrinsic nature of $\mathrm{Li}^{+}$ migration.

$$
D^{*}=\frac{1}{6} a^{2} v \exp \left(-\frac{E_{a}}{k_{B} T}\right)
$$

In equation (6), $\mathrm{E}_{\mathrm{a}}$ is the activation energy barrier for $\mathrm{Li}^{+}$migration, $v$ is the vibration frequency of migrating $\mathrm{Li}^{+}$in the lattice, $\mathrm{k}_{\mathrm{B}}$ is Boltzmann's constant, $\mathrm{T}$ is the absolute temperature, and a is the hopping distance [17].

\subsection{Ionic conductivity}

In electrochemical devices such as LIBs, ionic conductivity $(\sigma)$ refers to the movement of $\mathrm{Li}^{+}$under the influence of an external potential. The Nernst-Einstein equation describes the relationship between $\sigma$ and D:

$$
\frac{\sigma_{i}}{D_{i}}=\frac{Z_{i}^{2} F^{2} C_{i}}{R T}
$$

where,

$D_{i}=$ chemical diffusion coefficient of species $i$,

$\mathrm{R}=$ gas constant,

$\mathrm{T}=$ absolute temperature,

$\sigma_{\mathrm{i}}=$ ionic conductivity of species $\mathrm{i}$,

$\mathrm{Z}_{\mathrm{i}}=$ charge,

$\mathrm{F}=$ Faraday's constant, and

$\mathrm{C}_{\mathrm{i}}=$ concentration of species $\mathrm{i}$

$\mathrm{D}_{\mathrm{i}}$ can be related to ionic mobility through the Nernst-Planck equation. Equation (8) further illustrates this relationship:

$$
D_{i}=u_{i} k_{B} T
$$

where $u_{i}$ refers to the mobility for species I [17].

\subsection{Diffusion mechanisms}

Generally, microscopic diffusion is caused by the thermal energy of the material. Below the melting point, the amplitude of phonons at equilibrium is low, and the thermal energy plays a critical role in deviating the atoms from their mean positions by increasing the temperature. In an excited state, atoms tend to overcome the energy barrier, leading to atomic migration. However, some conditions must be satisfied before this atomic migration can occur. An adjacent empty site must be available, and the atom must possess certain energy to break neighboring bonds and cause lattice distortions, thereby leading to atomic migration.

A solid is never perfect, and there exist certain defects in the crystal lattice of solids. These defects exist in various forms within the solids, such as simple missing atoms (vacancies) and interstitial or substitutional impurities within the crystal lattice. The existence of defects not only governs the purity of the solid structure but also affects the material performance. Furthermore, the diffusion inside solids can be categorized into two types: vacancy assisted diffusion and non-vacancy assisted diffusion [22].

\subsubsection{Vacancy-assisted diffusion process}

The diffusion is governed by the perturbations of atoms in the equilibrium state. Vacancy-assisted diffusion occurs easily in the crystal lattice, as compared to non-vacancy assisted diffusion. In the vacancyassisted diffusion process, atoms are exchanged from a normal lattice site to the adjacent vacancy. When the atoms diffuse along a particular direction, the vacancy simultaneously moves in a direction oppo-

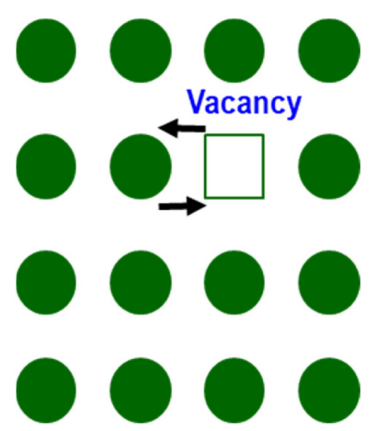

Fig. 6. Vacancy-assisted diffusion in a crystal lattice. The figure was reproduced with permission from Ref. [23]. Copyright 2003, Encyclopedia of Applied Physics. 
site to the atomic diffusion. Vacancy-assisted diffusion is directly related to the concentration of vacancies in the solid crystal lattices. When the vacancies are present in the crystal lattice, there is a relatively lower lattice strain during atomic hopping, and a lower activation energy barrier is experienced by the lattice atoms [22].

On charging LIBs, the removal of $\mathrm{Li}^{+}$creates vacancies in the crystal structure of active materials (Fig. 6). The change in the lattice structure is rather small during the de/intercalation of $\mathrm{Li}^{+}$in active materials, which is a crucial requirement for the improved and prolonged cycling performance of LIBs. The concentration of vacancies in the crystal structure plays a vital role in determining the pathway as well as the energy barrier for $\mathrm{Li}^{+}$migration. Fanghua et al. [24] showed that $\mathrm{LiCoO}_{2}$ exhibited varying energy barriers and $\mathrm{Li}^{+}$diffusion pathways due to the presence of monovacancies and divacancies.

\subsubsection{Non-vacancy assisted diffusion process}

As the lattice strain inside the crystal structure is elevated due to the absence of vacancies, nonvacancy assisted diffusion is more complicated. These interstitial atoms are occasionally impurities or solute atoms that are doped or mixed to alter material properties.

According to the direct interstitial diffusion mechanism, an interstitial atom moves to an adjacent site, and the lattice undergoes a significant amount of strain. However, there is no permanent strain in the lattice on the completion of the diffusion process, because the matrix atom displacement is temporary, as shown in Fig. 7(a). The sizes of the interstitial atoms are smaller than the sizes of the matrix atoms [22].

On the contrary, the knock-off migration mechanism represents an indirect interstitial mechanism wherein the size of the interstitial atom is equal to that of the matrix atom, as shown in Fig. 7(b). During the diffusion process, an interstitial atom pushes the matrix atom towards another interstitial site, inducing a large strain in the lattice. Chen et al. [25] stated that $\mathrm{Li}^{+}$diffusion is dominated by the knock-off diffusion mechanism during the lithiation of $\mathrm{Li}_{7} \mathrm{Ti}_{5} \mathrm{O}_{12}$.

(a)

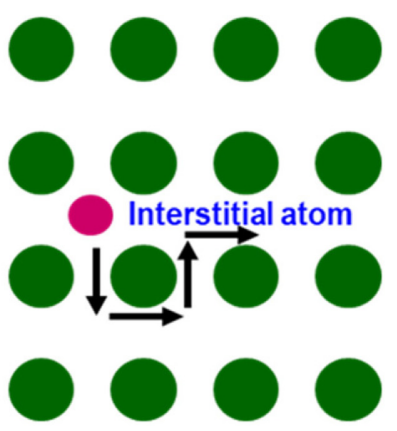

Knock-off Mechanism

(b)

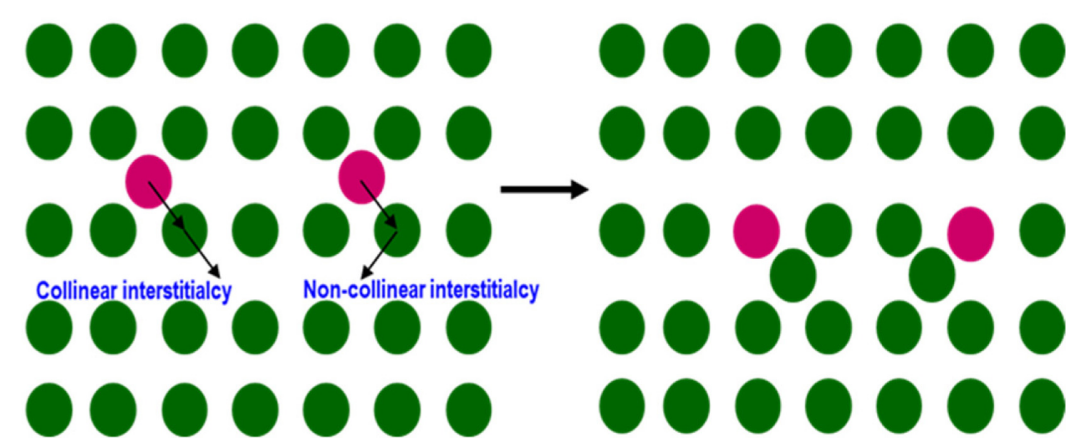

Fig. 7. (a) Direct interstitial diffusion in a crystal lattice. (b) Knock-off migration mechanism. The figure was reproduced with permission from Ref. [23]. Copyright 2003, Encyclopedia of Applied Physics. 


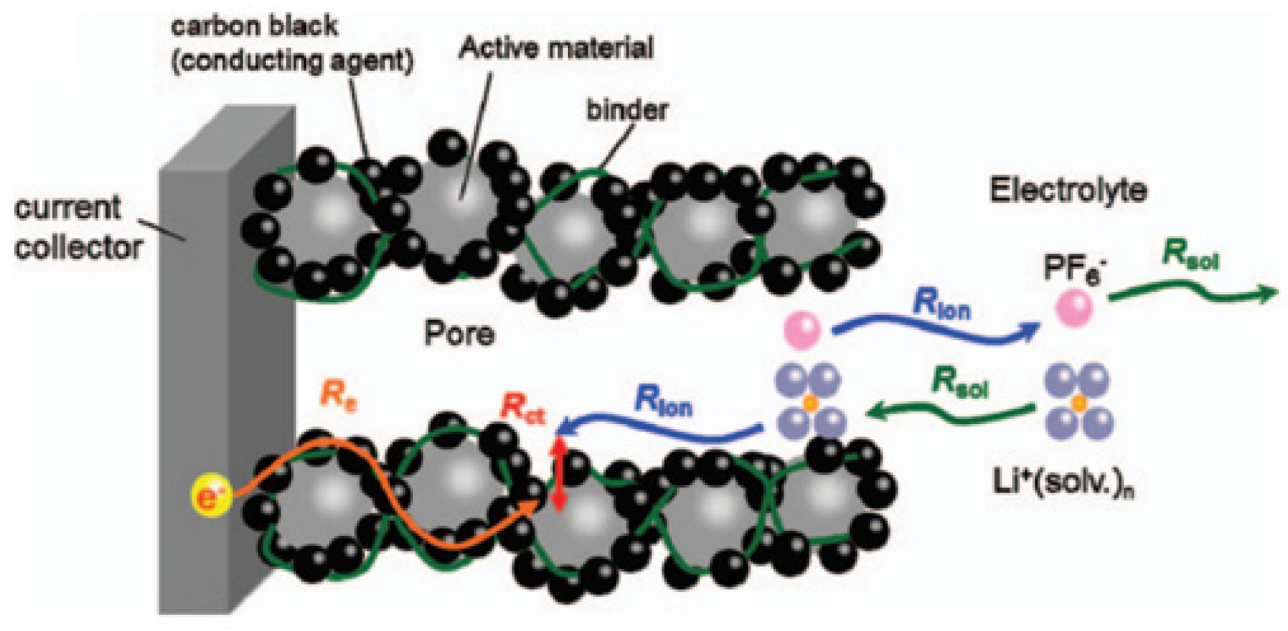

Fig. 8. Internal resistance in a porous electrode. The figure was reproduced with permission from Ref. [26]. Copyright 2012, Journal of Electrochemical Society.

\subsection{Ionic transport in a porous electrode}

The ionic transport in the electrolyte impregnated in the porous electrode is a predominant factor for determining the rate capability of LIBs. Ogihara et al. $[26,27]$ combined electrochemical impedance spectroscopy with a transmission line model theory for cylindrical pores, to understand the internal resistance inside electrolyte-infused porous electrodes.

As shown in Fig. 8, there are different types of internal resistance at the electrode-electrolyte interface. Moreover, the charge transfer resistance to $\mathrm{Li}^{+}$ and electron transport is dependent on the thickness of the electrode.

In the electrodes, ionic resistance in the pores $\left(\mathrm{R}_{\mathrm{ion}}\right)$ is affected by the pore length, whereas $R_{c t}$ is inversely proportional to the active surface area. $R_{\text {ion }}$ is mainly responsible for the $\mathrm{Li}^{+}$conduction in pores filled with the electrolyte. Hence, the power capability of LIBs is strongly affected by the ionic conduction pathways in electrodes [27].

Fukutsuka et al. [8] conducted a model study involving anodic nanoporous alumina featuring a porous framework with honeycomb-shaped pores ranging from 20 to $68 \mathrm{~nm}$. It was observed that the specific $\sigma$ of the electrolyte infused in the honeycomb pores was smaller than that of the bulk electrolyte solution (i.e., $7.6 \mathrm{mS} / \mathrm{cm}$ ), regardless of the pore size. The Nyquist plots exhibited depressed semi-circles, referring to the presence of ionic transport resistance in the honeycomb pores. The plots also indicated the existence of the capacitive element associated with the ionic transport inside the active material. Hence, the specific $\sigma$ of the electrolyte solution impregnated in the porous electrode influences the ionic transport resistance of the electrodes [8].

\subsection{Ion transfer at the interface between the active material and electrolyte}

The electrochemical kinetics at the electrodes of LIBs are affected by multiple factors. One of these factors is the charge transfer process involved at the electrode-electrolyte interface. Thus, it is also necessary to understand the reaction kinetics of the active materials. Abe et al. [28] evaluated the activation energy required for desolvation at the electrode-electrolyte interface.-The semi-circles in the Nyquist plots were influenced by the salt concentration of the electrolyte and the electrode potential. The resistance is inversely proportional to the salt concentration of the electrolyte. The activation energy values were calculated for the de-solvated and solvated $\mathrm{Li}^{+}$transfer based on the charge-transfer resistance vs temperature plots. The activation energy values for $\mathrm{Li}^{+}$ solvation were observed to be half of those required for the desolvation process [29].

On the contrary, solid electrolyte interphase (SEI) is a passive layer formed on the surface of the electrodes through the decomposition of electrolytes. The 


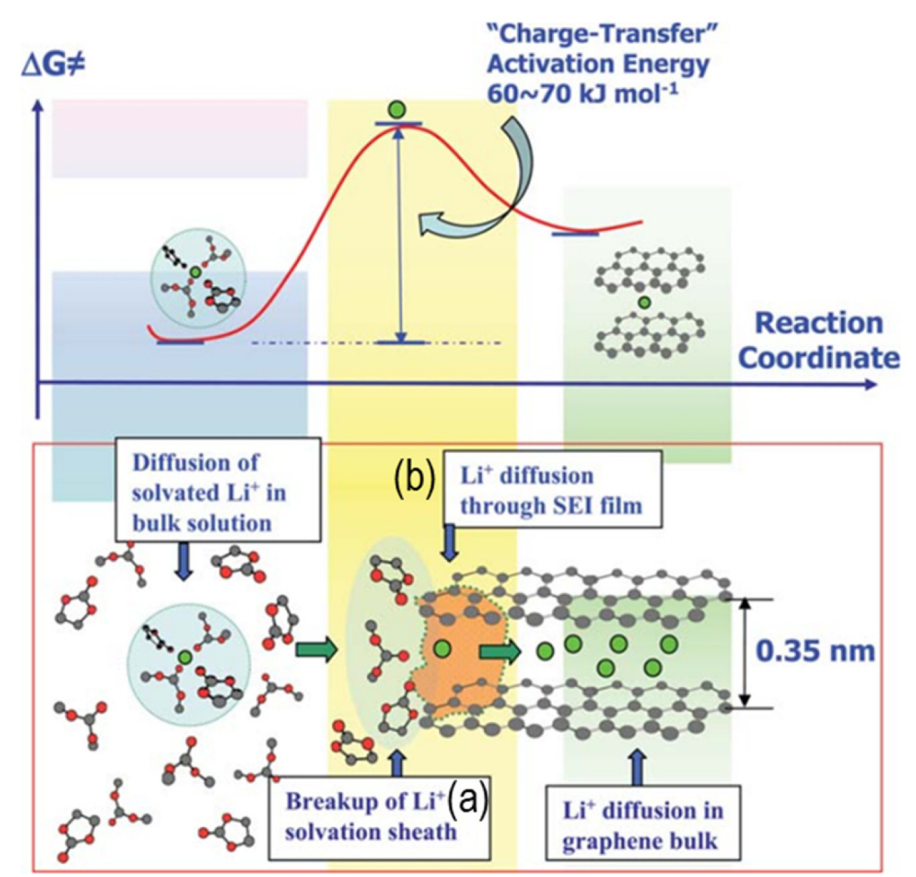

Fig. 9. Interphasial resistance from two sub-processes: (a) desolvation of $\mathrm{Li}^{+}$and (b) migration of naked $\mathrm{Li}^{+}$across the interphase. The figure was reproduced with permission from Ref. [11]. Copyright 2011, Journal of Material Chemistry.

interphases assist in the reversible $\mathrm{Li}^{+}$de/intercalation during the operation of LIBs, while restricting parasitic reactions.

$\mathrm{Xu}$ et al. [11] explained the interphasial reactions for solvation and desolvation. The solvation sheath of $\mathrm{Li}^{+}$is composed of the solvent molecules that bind together with the $\mathrm{Li}^{+}$core. These molecules are stabilized and remain undisplaced owing to the strong coulombic forces exerted by $\mathrm{Li}^{+}$on its surrounding molecules.

This sheath tends to stabilize $\mathrm{Li}^{+}$in the electrolyte by compensating the increase of Gibbs free energy. When $\mathrm{Li}^{+}$intercalates into the graphite anode, this solvation sheath must be stripped out because the SEI on the graphite surface only allows the intercalation of naked $\mathrm{Li}^{+}$. This corresponds to the existence of a high energy transition state, as shown in Fig. 9. The desolvation process induces an additional resistance to $\mathrm{Li}^{+}$transport and is a rate-determining step at the electrode-electrolyte interface [11].

\section{6 $\mathrm{Li}^{+}$diffusion in the active material}

Ionic diffusion inside the active materials plays a vital role in determining the performance of LIBs.
Ionic diffusion is generally favorable inside the battery; however, leakage current should be avoided. Since the rate capability of LIBs depends on the ionic charge transfer kinetics, it is important to understand the critical factors governing charge transfer, to evaluate the power characteristics of LIBs [30]. In LIBs, the $\mathrm{Li}^{+}$de/intercalation process begins with the dissociation of $\mathrm{Li}^{+}$-electron pairs and ends with the concomitant entry of $\mathrm{Li}^{+}$-electron pairs into active materials via solid-state diffusion [31]. Ionic diffusivity $\left(D_{\text {ionic }}\right)$, as expressed in equation $(9)$, is a parameter to characterize the ease of $\mathrm{Li}^{+}$movement in active materials:

$$
D_{\text {ionic }}=D_{0} \exp \left(-\frac{\Delta G}{k_{B} T}\right)
$$

A decrease in the energy barrier can increase $\mathrm{Li}^{+}$ diffusivity $\left(\mathrm{D}_{\mathrm{Li}}\right)$. Although an increase in operating temperature $(\mathrm{T})$ enhances diffusivity, it can pose a threat to safety.

$$
\tau d=\frac{\lambda^{2}}{D_{\text {ionic }}}
$$

$D_{\text {ionic }}$ at the surface must be enhanced to shorten 


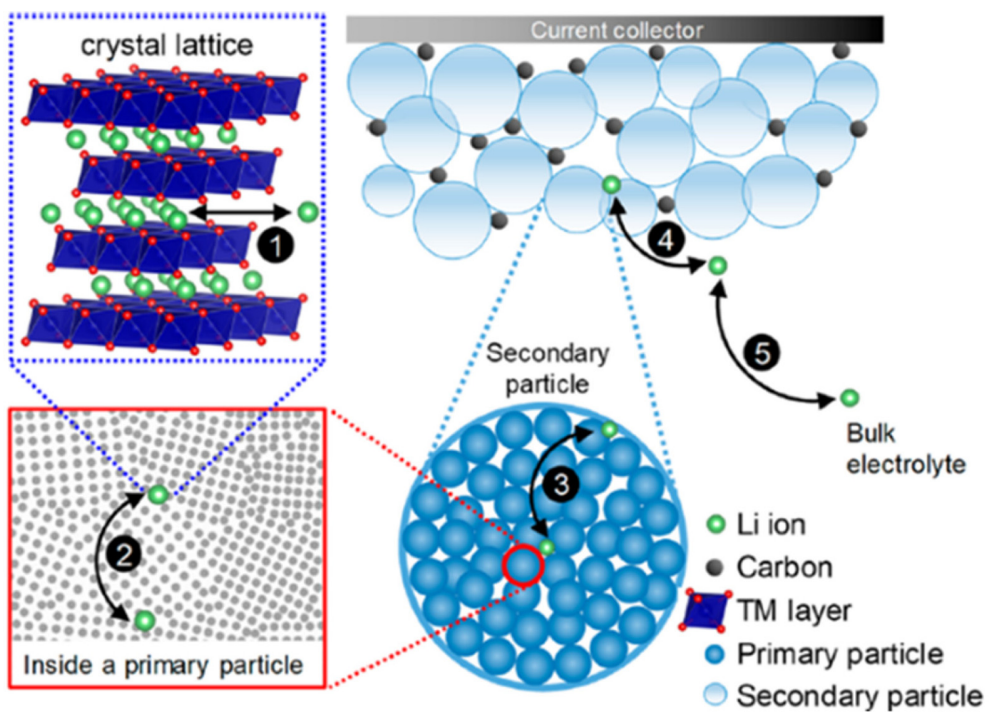

Fig. 10. Schematic diagram indicating $\mathrm{Li}^{+}$diffusion pathways. The figures were reproduced with permission from Ref. [32]. Copyright 2018, The Journal of Physical Chemistry Letters.

(a)

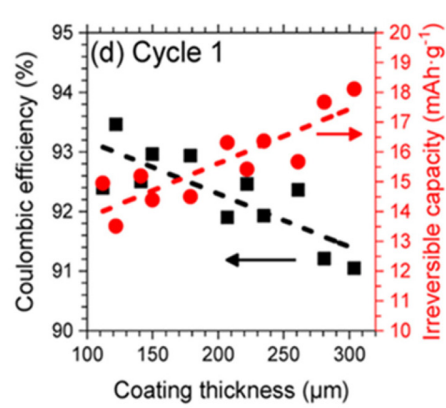

(b)

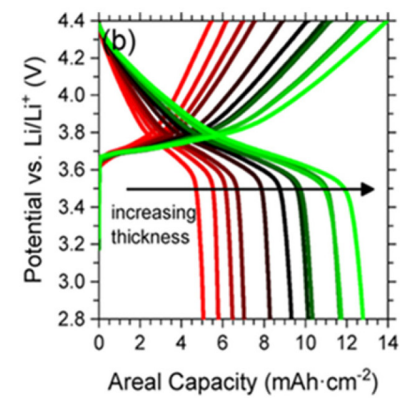

(c)

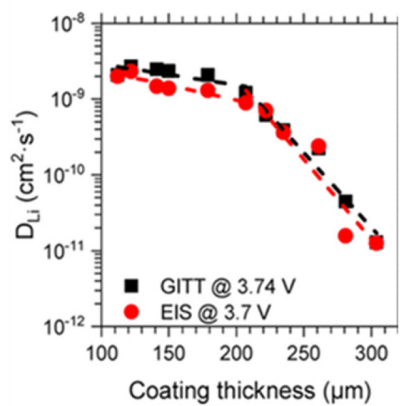

Fig. 11. (a) Coulombic efficiency and irreversible capacity of electrodes during the first cycle and (b) voltage profiles with varying thickness. (c) $\mathrm{Li}^{+}$diffusivity. The figures were reproduced with permission from Ref. [32]. Copyright 2018, The Journal of Physical Chemistry Letters.

the diffusion time ( $\tau \mathrm{d})$ expressed by equation (10), and the electron/ion diffusion length $(\lambda)$ should be reduced [31]. The following schematic describes the diffusion pathways of $\mathrm{Li}^{+}$within the active material.

As depicted in Fig. 10, during $\mathrm{Li}^{+}$diffusion in the electrode, the ions sequentially move through different parts of the electrode, namely the crystal structures, primary particles, secondary particles, porous electrodes, and bulk electrolyte [32]. Although the energy density of an LIB can be improved by increasing the thickness of the electrode, the $\mathrm{Li}^{+}$kinetics is significantly reduced.
As shown in Fig. 11(a-b), the thicker electrodes exhibited a significant capacity loss coupled with a low coulombic efficiency. $\tau \mathrm{d}$ is a function of electrode thickness; therefore, the time required for $\mathrm{Li}^{+}$ diffusion increased with an increase in electrode thickness. $\mathrm{D}_{\mathrm{Li}}$ also depends on electrode thickness and directly affects the specific capacity and rate capability of LIBs (Fig. 11(c)). During the operation of LIBs, a rapid decrease in $\mathrm{D}_{\mathrm{Li}}$ was observed for thicker electrodes due to the localized oversaturation or over-depletion of $\mathrm{Li}^{+}$induced by the slower $\mathrm{Li}^{+}$ transport [32]. 
(a)
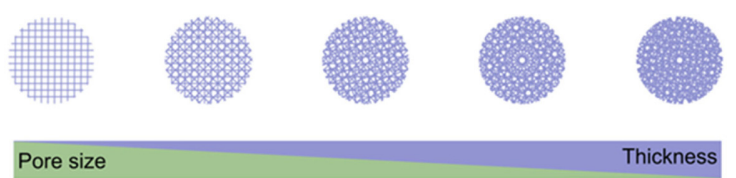

(b)

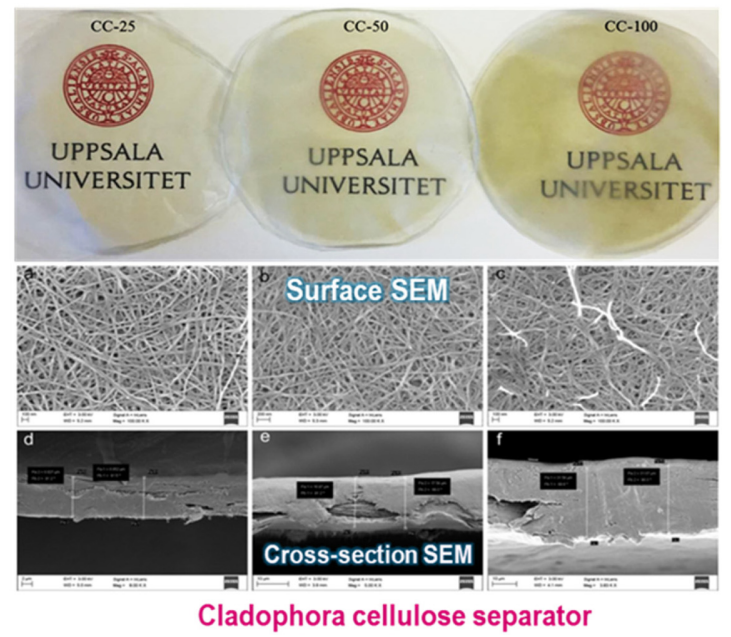

Fig. 12 (a) Dependency of the pore size on the thickness of the separator. (b) Digital images, (c) surface SEM, and (d) cross-section SEM images of Cladophora cellulose separator. The figures were reproduced with permission from Ref. [34]. Copyright 2017, Cellulose.

Table 1. Properties of Cladophora cellulose (CC) separators. The table was reproduced with permission from Ref. [34]. Copyright 2017, Cellulose.

\begin{tabular}{ccccc}
\hline \hline & Thickness $(\mu \mathrm{m})$ & Pore size $(\mathrm{nm})$ & Porosity $(\%)$ & $\sigma(\mathrm{mS} / \mathrm{cm})$ \\
\hline CC-25 & 10 & 21 & 44 & 0.82 \\
CC-50 & 20 & 13 & 37 & 0.79 \\
CC-100 & 40 & 12 & 33 & 0.69 \\
\hline
\end{tabular}

\subsection{Ion transport in the bulk electrolyte through the separator}

Thin separators are more suitable for enhancing the performance of LIBs, because thick separators are not feasible owing to their poor ionic conductivity. A thin separator typically possesses high porosity, which enables enhanced mass transport through the pores with reduced resistance from the electrolyte. Thus, the porosity of separators is a crucial factor in determining the internal resistance of LIBs. A uniform porosity of separators is desirable for maintaining a uniform current density with a smooth ionic current flow inside the LIBs. A non-uniform current density can cause a significant reduction in performance [33].
As the resistance is also affected by the pore structure of the separators, the total pore volume and pore size of separators could be tailored to enhance $\sigma$ by decreasing the thickness of the separators (Fig. 12). A thinner separator enables an increase in the mass transportation of ions, thereby effectively lowering the resistance even at high current densities. For improving cycling performance and rate capability, the separator should be as thin as possible to minimize the cell resistance but still be able to maintain high ionic conductivity.

Pan et al. [34] demonstrated that small over-potential and high specific capacity of $\mathrm{LiFePO}_{4}$ could be attained by employing a thin separator even at high current densities. 

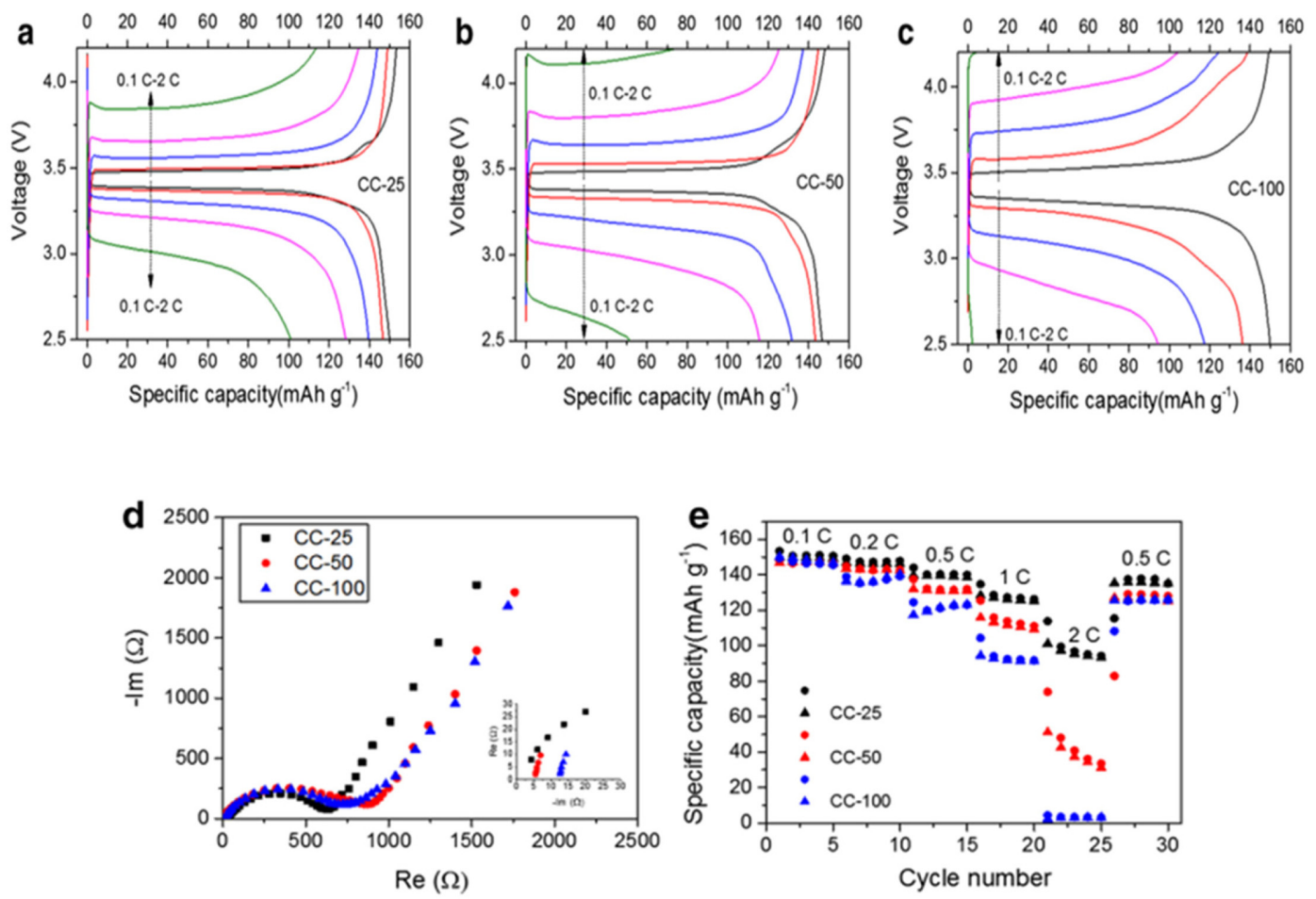

Fig. 13 Charge-discharge curves at various cycling rates for the (a) CC-25, (b) CC-50, and (c) CC-100 based cells. (d) Nyquist and (e) capacity retention plots of Cladophora cellulose (CC) separators. The figures were reproduced with permission from Ref. [34]. Copyright 2017, Cellulose.

As shown in Table 1 and Figs. 12 and 13(a-c), the lowest cell resistance was observed for Cladophora cellulose (CC)-25 separator with the highest porosity and lowest thickness. This supports the hypothesis that a reduction in the ionic transport path is beneficial for lowering cell resistance. As shown in Fig. 13(d-e), the cycling performance of CC-25 is superior to other samples due to the lower cell resistance.

\section{Material Engineering}

The material design is the most crucial step when developing high-performance LIBs.

Structural modifications of active materials are effective methods to reduce the interfacial resistance during electrode processes. Some of the effective structural modifications of active materials, depending on the type of active materials and interface scenarios, for improving the electrochemical performance of LIBs are briefly explained in the following section.

\subsection{Surface modifications}

Li-rich cathode materials, commonly known as over-lithiated oxides (OLOs), have received significant attention as promising cathode materials for LIBs. In particular, lithium manganese oxide $\left(\mathrm{Li}_{2} \mathrm{MnO}_{3}\right)$, which has a monoclinic structure of the $\mathrm{C} 2 / \mathrm{m}$ space group, can offer a high capacity of $300 \mathrm{mAh} / \mathrm{g}$ on an initial charge to $4.8 \mathrm{~V}$ vs $\mathrm{Li} / \mathrm{Li}^{+}$. However, the practical use of $\mathrm{Li}_{2} \mathrm{MnO}_{3}$ is limited due to the low coulombic efficiency and irreversible capacity loss during the first cycle. Kim et al. [35] reported a positive effect of the $\mathrm{TiO}_{2}$ surface coating on $\mathrm{Li}_{2} \mathrm{MnO}_{3}$, where $\mathrm{TiO}_{2}$ acts as a suitable alternative for improving the structural stability of $\mathrm{Li}_{2} \mathrm{MnO}_{3}$. $\mathrm{TiO}_{2}$-coated $\mathrm{Li}_{2} \mathrm{MnO}_{3}$ exhibited a relatively lower interfacial resistance, as compared with that of uncoated $\mathrm{Li}_{2} \mathrm{MnO}_{3}$. TiO ${ }_{2}$ acted as a stabilizer and prevented the decomposition of the active material in the electrolyte at high potentials, thereby improving the rate performance of $\mathrm{TiO}_{2}$-coated $\mathrm{Li}_{2} \mathrm{MnO}_{3}$. $\mathrm{LiCoO}_{2}$ has an $\alpha-\mathrm{NaFeO}_{2}$ layered structure, which assists in 
(a)

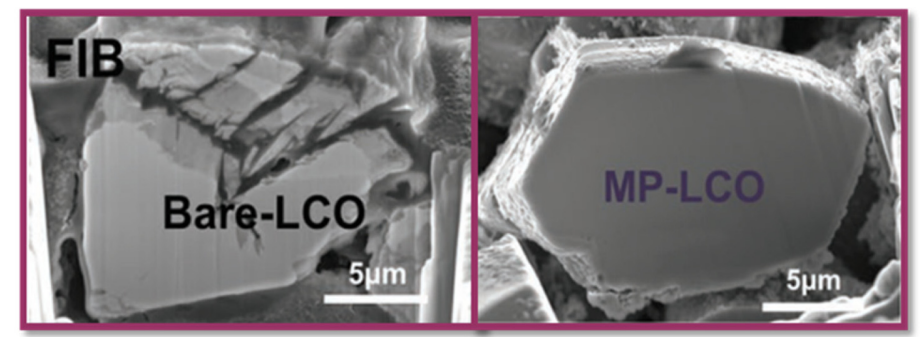

(b)

(c)
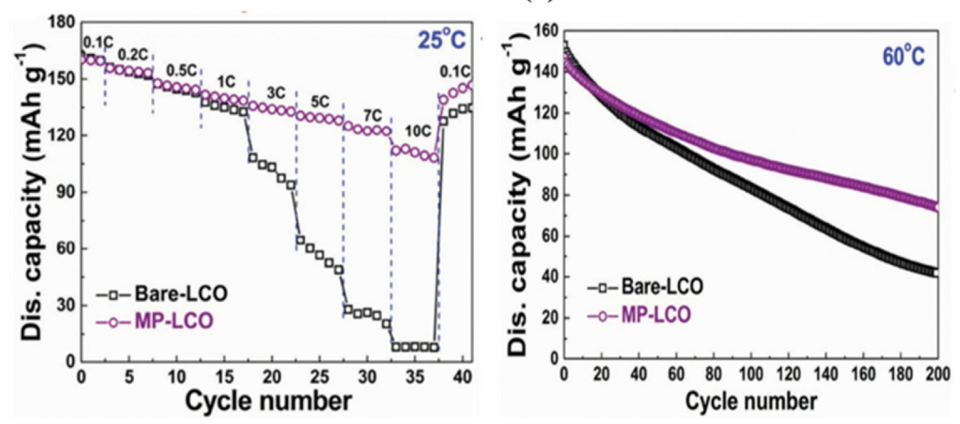

Fig. 14. (a) FIB micrographs of bare and $\mathrm{MP}$-coated $\mathrm{LiCoO}_{2}$ particles. Cycling stability of bare and MP-coated $\mathrm{LiCoO}_{2}$ at (a) $25^{\circ} \mathrm{C}$ and (b) $60^{\circ} \mathrm{C}$. The figures were reproduced with permission from Ref. [36]. Copyright 2017, Advanced Energy Materials.

efficient reversible $\mathrm{Li}^{+}$intercalation chemistry. Despite its high theoretical capacity, the working capacity of $\mathrm{LiCoO}_{2}$ is limited to just $140 \mathrm{mAh} / \mathrm{g}$. This limited capacity of $\mathrm{LiCoO}_{2}$ originates from a factual phase transition (at $>4.2 \mathrm{~V}$ ) from a hexagonal to a monoclinic structure, causing a decrease in the coulombic efficiency. This leads to a decrease in $\mathrm{Li}^{+}$diffusion, resulting in high internal strain and degradation of mechanical properties.

A large number of $\mathrm{LiCoO}_{2}$ surface coatings have been explored to overcome these problems. In particular, surface coatings containing metallic compounds were found to be effective because they act as a barrier between the electrolyte and the $\mathrm{LiCoO}_{2}$ particles. Kaluri et al. [36] reported a hybrid surface coating consisting of magnesium $(\mathrm{Mg})$ and phosphorous $(\mathrm{P})$ on the surface of $\mathrm{LiCoO}_{2}$.

A surface coating consisting of $\mathrm{Mg}$ and $\mathrm{P}$ can be effective for improving the electrochemical performance and thermal stability of $\mathrm{LiCoO}_{2}$ (Fig. 14(a)). After surface coating, $\mathrm{Mg}$ and $\mathrm{P}(\mathrm{MP})$-coated $\mathrm{LiCoO}_{2}$ exhibited a high-capacity retention of $86.5 \%$, even after 100 cycles. As shown in Fig. 14(b-c), the rate capability of MP-coated $\mathrm{LiCoO}_{2}$ was nearly 25 times higher than the rate capability of bare $\mathrm{LiCoO}_{2}$, even at a high current density of $10 \mathrm{C} \mathrm{[37].}$

The decrease in the capacity of bare $\mathrm{LiCoO}_{2}$ cathode could be attributed to the structural degradation at high operating temperatures [36]. As a result, a significant dissolution of Co in the electrolyte was observed based on FIB post mortem analyses (Fig. 14(a)).

In addition, post-processes (i.e., heat-treatment and washing) can improve the rate performance of nickel (Ni)-rich layered materials. The surface of a treated, Ni-rich layered material is considerably cleaner than that of an untreated material, as shown in Fig. 15. As a result, treated cathodes exhibit a significantly higher rate performance than untreated materials due to the reduction in surface contamination [38].

\subsection{Size control}

\subsubsection{Particle size effect}

Nanostructured materials typically possess large surface areas as compared to bulk materials. Thus, it should be noted that reducing the particle size would be a wise strategy for increasing the surface area of active materials. The properties of active materials 


\section{Ni-rich layered material}

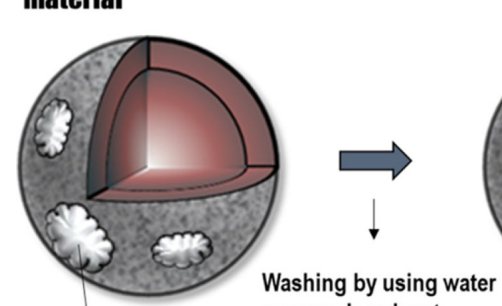
or organic solvent

$\checkmark$

Lithium compounds $\left(\mathrm{Li}_{2} \mathrm{CO}_{3}, \mathrm{LiOH}\right.$ and etc.)
A vulnerable surface

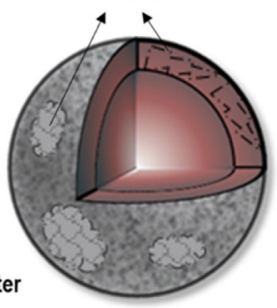

Reduction in surface contamination

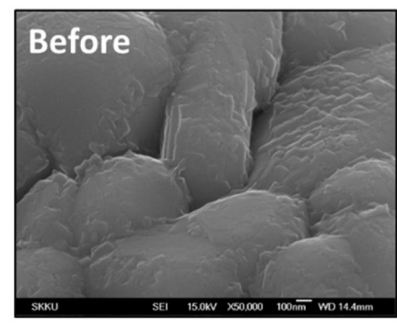

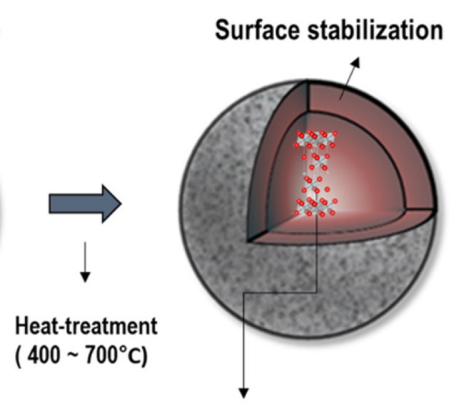

Bulk crystal structure change

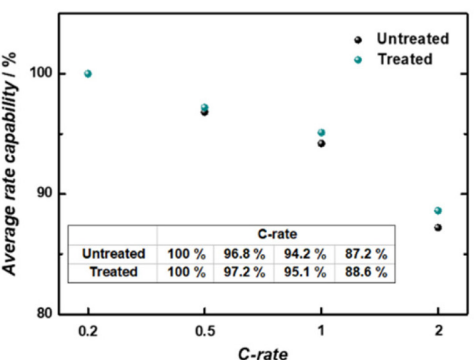

Fig. 15. Schematic of post-processes (washing and heating) of Ni-rich layered cathode materials, SEM images for untreated (before) and treated (after) materials, and rate performances. The figures were reproduced with permission from Ref. [38]. Copyright 2020, Journal of Materials Chemistry A.

change drastically at the nanometer scale because the active surface area is enlarged. In LIBs, the diffusion distance and $\mathrm{D}_{\mathrm{Li}}$ are correlated to the particle size of active materials, which directly affects the movement of $\mathrm{Li}^{+}$inside the active materials of electrodes [39].

Jiang et al. [40] studied the effect of the particle size of anatase $\mathrm{TiO}_{2}$ on the electrochemical performance of active materials. A decrease in the particle size of anatase $\mathrm{TiO}_{2}$ reduces the interstitial sites for $\mathrm{Li}^{+}$insertion, resulting in a reduced diffusion or transport length of $\mathrm{Li}^{+}$; this improves the rate capability. Anatase $\mathrm{TiO}_{2}$ featuring a particle size of $6 \mathrm{~nm}$ exhibited a more stable cycling performance, as compared to other materials. Thus, a reduction in particle size increases the active surface area of active materials and facilitates the de/intercalation of $\mathrm{Li}^{+}$, thereby leading to an improvement in the rate capability as well as the specific capacity [40].

\subsubsection{SEI Thickness}

Liu et al. [41] demonstrated the enhanced performance of LIBs by employing chemically active multifunctional separators (MFSs). From a microscopic perspective, the capacity retention of electrodes can be increased through a simultaneous reduction in the interfacial resistance. MFSs would suppress parasitic reactions at the interfaces and reduce the SEI film thickness on electrodes, leading to a reduction in the interfacial resistance.

The cycling performance and capacity retention of LIBs could be improved through the lower interfacial resistance achieved by a reduction in the SEI film thickness. A thin SEI film enables a homogeneous distribution of current on the electrode surface, leading to an improved performance of the LIB.

\subsection{Synergetic effect of the conductive binder}

As a commercial binder, PVDF is commonly used for electrode fabrication owing to its excellent electrochemical stability and binding property. However, the insulator PVDF induces an increase in the internal resistance of the electrodes. Ling et al. [42] incorporated naturally occurring conductive binder sodium alginate (SA) along with poly(3,4-propylenedioxythiopene) (PProDOT) in electrodes, as shown in Fig. 16 (a). 
(a)

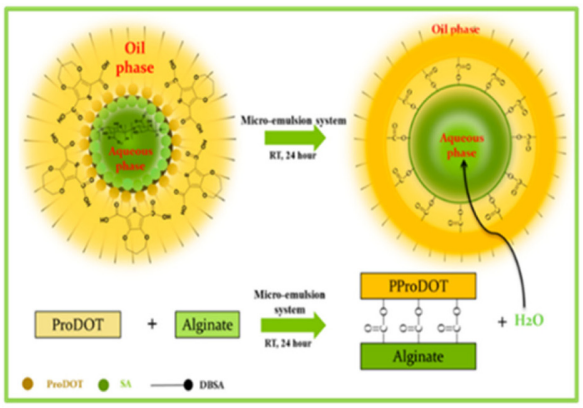

(c)

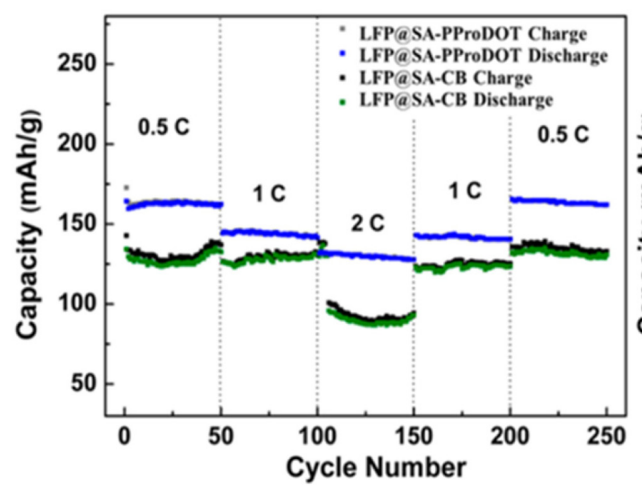

(b)

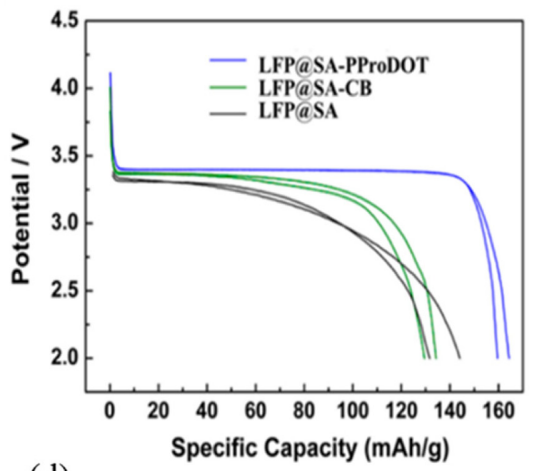

(d)

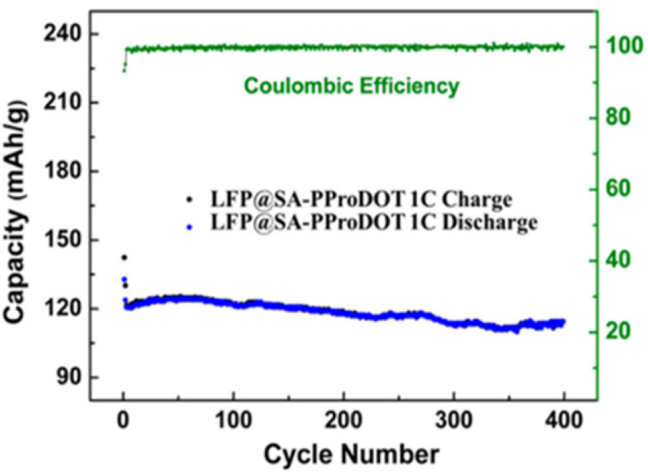

Fig. 16. (a) Micro-emulsion system for SA-PProDOT and (b) galvanostatic discharge profiles of $\mathrm{LiFePO}_{4}$ with different binders. (c) Rate performance of $\mathrm{LiFePO}_{4}$ and (d) $\mathrm{LiFePO}_{4}$ performance for different types of binders. The figures were reproduced with permission from Ref. [42]. Copyright 2015, Nano Letters.

As shown in Fig. 16(b), the electrochemical performance of $\mathrm{LiFePO}_{4}$ could be improved by employing the conductive SA-PProDOT binder. The cycling performance and capacity retention of $\mathrm{LiFePO}_{4}$ are depicted in Fig. 16 (c-d). The capacity retention of $\mathrm{LiFePO}_{4}$ with the SA-PProDOT binder was estimated to be $86.6 \%$, without a significant capacity loss even at a high current density of $1 \mathrm{C}$. Moreover, excellent Coulombic efficiency was noticeable even after 400 cycles. This clearly indicated that the conductive SA-PProDOT binder would be beneficial for improving electrochemical performance owing to its conductive nature [42].

\subsection{Nano-structuring}

Nano-structuring is useful for tailoring active materials so as to obtain desirable morphologies and microstructures such as particles, wires, sheets, and hollow porous structures. A short diffusion length and large surface area of nanostructured active materials could enhance the rate of ionic and electronic transport at the electrode-electrolyte interface. At the nanoscale, materials tend to have a large surface area, which is responsible for facilitating electrochemical reactions at the interfaces inside electrodes. Nanostructured materials lead to a decrease in the thermodynamic activation energy barrier at the electrodeelectrolyte interface $[31,43]$. For example, LTO is a commonly used active material featuring a high capacity and a suitable potential window. However, due to its low conductivity, the insertion of $\mathrm{Li}^{+}$in bulk LTO is limited. However, this could be further enhanced by decreasing the crystallite or particle size via nano-structuring. Feckl et al. [44] reported that $\mathrm{Li}^{+}$insertion is greater in nano-LTO than in porous LTO. 
As shown in Fig. 17, nano-LTO-400 with a particle size of $3 \mathrm{~nm}$ exhibited a better cycling performance, as compared to nano-LTO-500 with a particle size of
$6 \mathrm{~nm}$. Such an improvement in the performance can be explained by the enhanced insertion of $\mathrm{Li}^{+}$ induced by the smaller crystallite size [44]. (a)

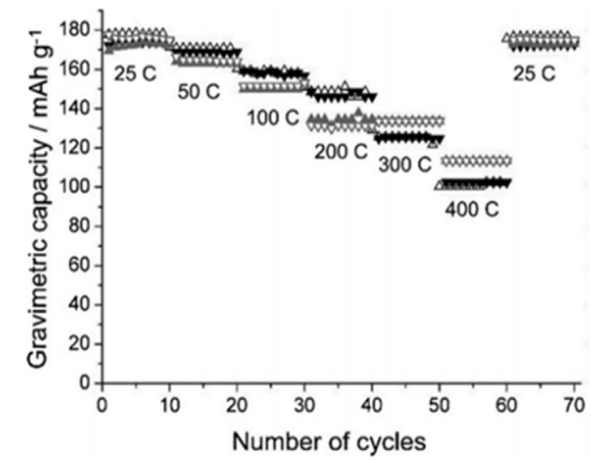

(b)

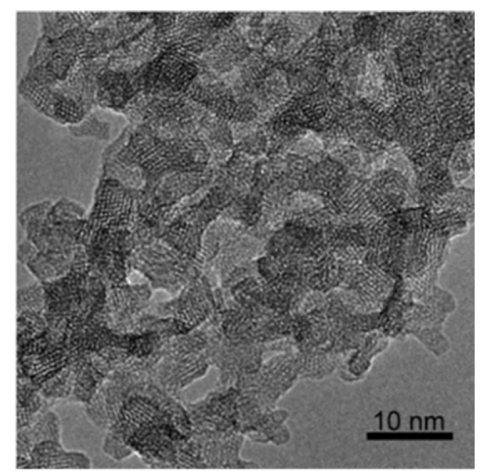

Fig. 17. (a) Multi-cycling stability of nano-LTO-400 (gray symbol) and nano-LTO-500 (black symbol) at different rates and (b) HR-TEM image of nano-sized LTO at $400^{\circ} \mathrm{C}$. The figures were reproduced with permission from Ref. [44]. Copyright 2012, Angewandte Chemie.

\section{N-Doped Graphene Electrode}

a

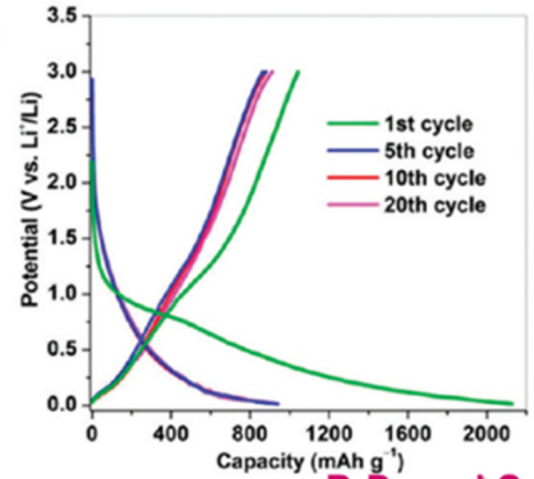

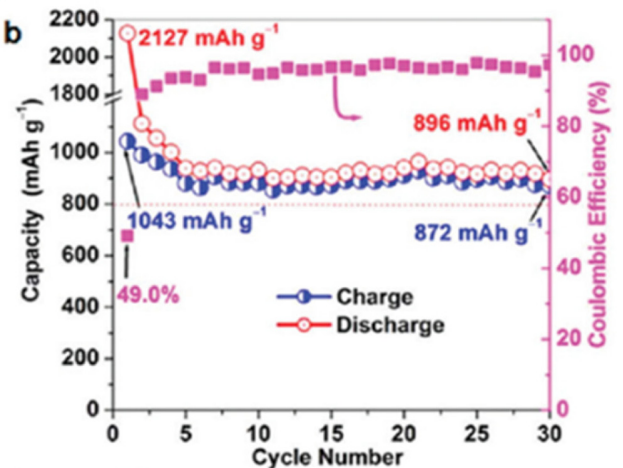

B-Doped Graphene Electrode

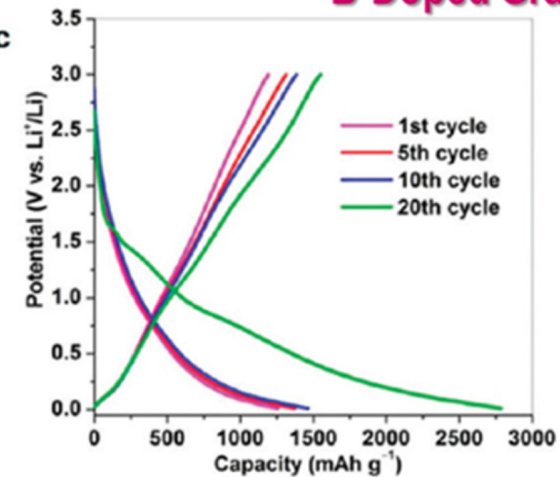

Fig. 18. Electrochemical performance of $(a, c)$ of N- and B-doped graphene electrode and (b, d) coulombic efficiency and cycle performance of $\mathrm{N}$ - and $\mathrm{B}$-doped graphene electrode $\mathrm{vs}^{+} \mathrm{Li}^{+} / \mathrm{Li}$. The figures were reproduced with permission from Ref. [45]. Copyright 2011, ACS Nano. 


\subsection{Dopant manipulation}

Simultaneously achieving high energy and high power at robust charging and discharging rates is a crucial requirement for the development of LIBs. Currently, LIBs still suffer from low power densities due to the use of bulk materials as active materials. Although bulk metal oxides or graphite anodes can offer high energy densities, the power density achieved is limited by the slow kinetics in bulk materials. The power characteristics of LIBs are dependent on efficient charge storage capability and also on the kinetics of charge transport during electrochemical reactions in the bulk electrode and electrolyte. Doping or substitution in bulk materials is an effective approach to introduce ions, heteroatoms, or vacancies in the crystal structure; this in turn enhances conductivity due to the excess or deficiency of charged species, leading to an improvement in the overall performance of the battery. Wu et al. [45] reported the substitution of nitrogen $(\mathrm{N})$ and boron (B) atoms in place of carbon (C) atoms on the edges of graphite. $\mathrm{B}$ and $\mathrm{N}$ doping in graphene facilitates $\mathrm{Li}^{+}$intercalation into the host structure of graphite and enhances electrical conductivity, thereby leading to high power and energy densities.

Graphene exhibited an enhanced coulombic efficiency during the cycles, after $\mathrm{N}$ and $\mathrm{B}$ doping, as shown in Fig. 18. The reversible capacity retentions for $\mathrm{N}$-doped and B-doped graphene were measured to be $83.6 \%$ and $79.2 \%$, respectively. The improvements in the parameters indicated the effective suppression of side reactions and electrolyte decomposition as well as the enhancement in $\mathrm{Li}^{+}$absorption and the diffusion inside electrodes [45].

In addition, the $\mathrm{Li}^{+}$diffusion path length in the $2 \mathrm{D}$ layered system can be controlled through the dopant ratio. For instance, the inter-slab size of $\mathrm{LiO}_{6}$ in the Nirich layered material can be increased by reducing the amount of Mn ions (Fig. 19). Consequently, this leads to a favorable environment for $\mathrm{Ni}^{2+}$ to diffuse in the $\mathrm{Li}$ layer (i.e., lowering the activation energy). Therefore, NCM721 exhibits a significantly higher $\mathrm{D}_{\mathrm{Li}}$ than NCM523 [46].
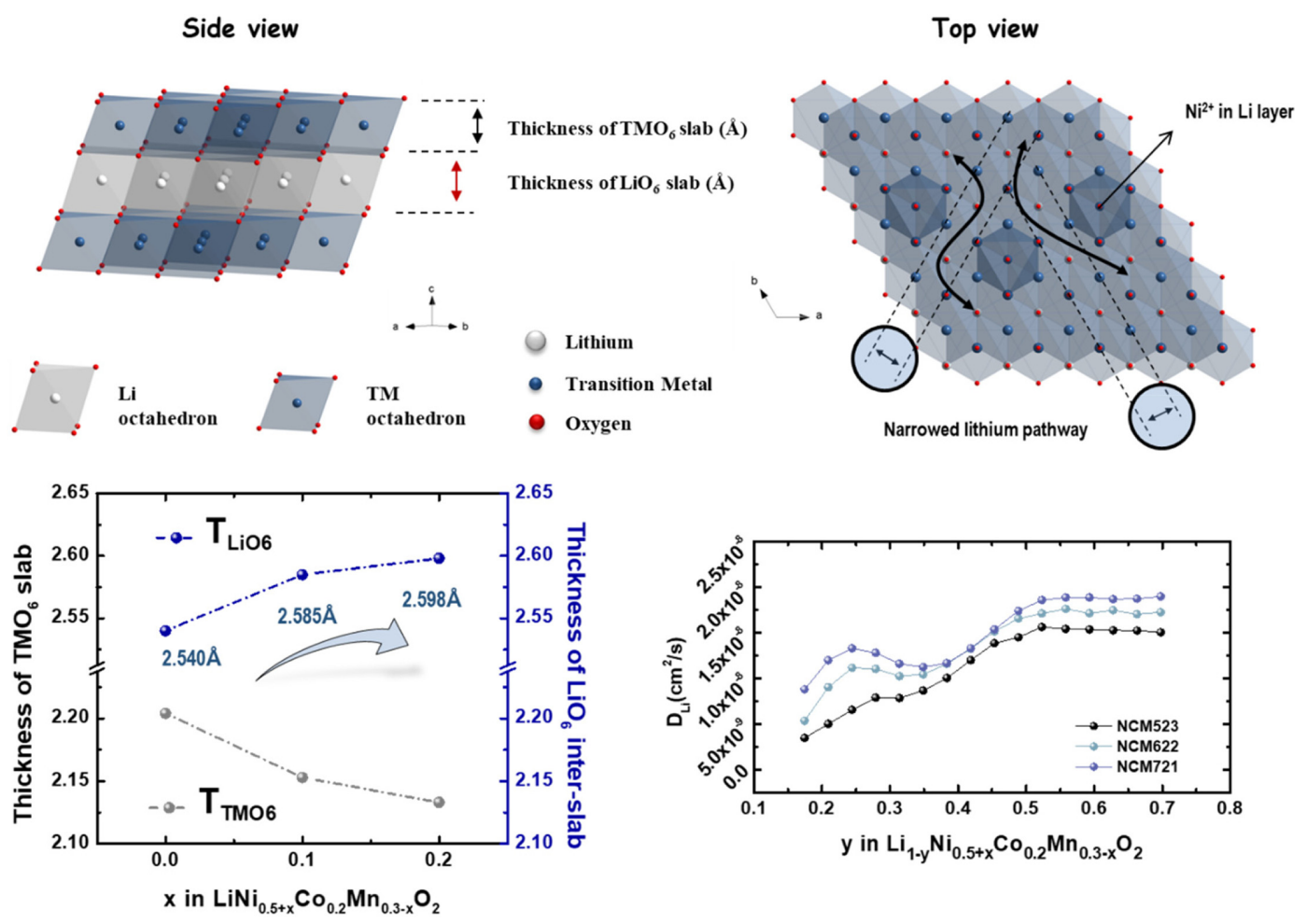

Fig. 19. Illustration of $\mathrm{LiNi}_{0.5+\mathrm{x}} \mathrm{Co}_{0.2} \mathrm{Mn}_{0.3-\mathrm{x}} \mathrm{O}_{2}(\mathrm{x}=0,0.1$, and 0.2$)$ layered structure, slab thickness, and diffusion coefficients of each material during charging. The figures were reproduced with permission from Ref. [46]. Copyright 2017, Advanced Energy Materials 


\subsection{Hybrid composite design}

A hybrid composite approach involves the synthesis of various composite materials by integrating conducting additives into active materials. The conducting additives induce the formation of sufficient conduction pathways in the electrodes. In addition to increasing the electrical conductivity, these conducting additives tend to decrease the length of $\mathrm{Li}^{+}$diffusion, enabling faster $\mathrm{Li}^{+}$de/intercalation.

Magasinski et al. reported a porous silicon ( $\mathrm{Si}$ )-C composite containing irregular open channels that facilitate the adsorption and diffusion of $\mathrm{Li}^{+}$within the composite material. Si typically undergoes a large volume expansion; however, in this case, the volume expansion of Si could be compensated by integrating free spaces in the composite. The Si-C composite exhibited better capacity retentions at high current densities, compared with those of simply annealed carbon black [47]. Thus, hybrid composites with a conducting additive could be a practical approach for further improving the electrochemical performance of the active materials in LIBs.

\section{Conclusions}

Currently, LIBs are associated with a major challenge that can only be overcome through a comprehensive understanding of the processes occurring at the electrode-electrolyte interface. Advanced materials can be efficiently designed only after obtaining an in-depth understanding of multiple electrode processes and interfacial reactions occurring at the electrodes. Conducting species always follow the path that offers the minimum transport resistance. Thus, the internal resistance to the transport of charged species depends on multiple intrinsic and extrinsic factors of the electrodes. The porous structure of the electrodes provides abundant interconnected open pores that enable electrolyte absorption, which considerably decreases the diffusion path of $\mathrm{Li}^{+}$. Understanding the basic scientific principles governing $\mathrm{Li}^{+}$ diffusion and electron transport in electrodes can help accelerate the pace of future research on improving the performance of LIBs. Reducing the resistance to the transport of charged species in electrodes would prove to be beneficial for achieving faster kinetics and enhanced electrochemical performance in the future.

\section{Acknowledgement}

This work was supported by the National Research Foundation of Korea (NRF) grant funded by the Korea government (MSIP) (No. NRF2019R1A2C2003731).

\section{References}

[1] M. Wang, Y. Tian, W. Liu, R. Zhang, L. Chen, L. Yinda, L. Xin, J. Clean. Prod., 2020, 265, 121769.

[2] L. Zhang, X. Qin, S. Zhao, A. Wang, J. Luo, Z. L. Wang, F. Kang, Z. Lin, B. Li, Adv. Mater, 2020, 33, 1908445.

[3] Q. Zhang, N. Cui, Y. Li, B. Duan, C. Zhang, J. Energy Storage., 2020, 27, 100945.

[4] Z. Chen, J. Lu, B. Liu, N. Zhou, and S. Li, Energies, 2020, 13(10), 2543.

[5] Y. Orikasa, Y. Gogyo, H. Yamashige, M. Katayama, K. Chen, T. Mori, K. Yamamoto, T. Masese, Y. Inada, T. Ohta, Z. Siroma, S. Kato, H. Kinoshita, H. Arai, Z. Ogumi, Y. Uchimoto, Sci. Rep., 2016, 6, 26382.

[6] Y. Itou, N. Ogihara, S. Kawauchi, J. Phys. Chem. C., 2020, 124(10), 5559-5564.

[7] A. Chu, A. Allam, A. Cordoba Arenas, G. Rizzoni,S. Onori, J. Power Sources, 2020, 478, 228991.

[8] T. Fukutsuka, K. Koyamada, S. Maruyama, K. Miyazaki, T. Abe, Electrochim. Acta,. 2016, 199, 380387.

[9] A. Yano, K. Hikima, J. Hata, K. Suzuki, M. Hirayama, R. Kanno, J. Electrochem. Soc., 2018, 165(14), A3221.

[10] X. Yu, A. Manthiram, Energy Environ. Sci., 2018, 11(3), 527-543.

[11] K. Xu, A. Von Cresce, J. Mater. Chem., 2011, 21(27), 9849-9864.

[12] M. Park, X. Zhang, M. Chung, G.B. Less, A.M. Sastry, J. Power Sources., 2010, 195(24), 7904-7929.

[13] C.J. Bae, C.K. Erdonmez, J.W. Halloran, Y.M. Chiang, Adv. Mater, 2013, 25(9), 1254-1258.

[14] D. Kehrwald, P.R. Shearing, N.P. Brandon, P.K. Sinha, S.J. Harris, J. Electrochem. Soc., 2011, 158(12), A1393.

[15] Y. Ren, A.R. Armstrong, F. Jiao, P.G. Bruce, J. Am. Chem. Soc., 2010, 132(3), 996-1004.

[16] B. Vijayaraghavan, D.R. Ely, Y.-M. Chiang, R. GarcíaGarcía, R.E. García, J. Electrochem. Soc., 2012, 159(5), A548.

[17] R.E. Hummel, Electronic Properties of MaterialsSpringer-Verlag., 2011.

[19] H.L. Pan, Y.S. Hu, H. Li, L.Q. Chen, Chinese Phys. B., 2011, 20(11), 118202.

[20] G.F. Yang, K.Y. Song, S.K. Joo, J. Mater. Chem. A., 2014, 2(46), 19648-19652.

[21] S.W. Oh, S.-T. Myung, H.J. Bang, C.S. Yoon, K. Amine, Y.-K. Sun, Electrochem. Solid-State Lett., 2009, 12(9) , A181. 
[22] H. Mehrer, Diffusion in Solids: Fundamentals, Methods, Materials, Diffusion-Controlled Processes, SpringerVerlag Berlin Heidelberg., 2003, 1-651.

[23] A. V. Chadwick, Encycl. Appl. Phys. 2003, 193-222.

[24] F. Ning, S. Li, B. Xu, C. Ouyang, Solid State Ionics 2014, 263, 46-48.

[25] Y.C. Chen, C.Y. Ouyang, L.J. Song, Z.L. Sun, Electrochim. Acta., 2011, 56(17), 6084-6088.

[26] N. Ogihara, S. Kawauchi, C. Okuda, Y. Itou, Y. Takeuchi, Y. Ukyo, J. Electrochem. Soc., 2012, 159(7), A1034.

[27] N. Ogihara, Y. Itou, T. Sasaki, Y. Takeuchi, J. Phys. Chem. C., 2015, 119(9), 4612-4619.

[28] T. Abe, H. Fukuda, Y. Iriyama, Z. Ogumi, J. Electrochem. Soc. 2004, 151(8), A1120.

[29] Z. Ogumi, Electrochemistry, 2010, 78(5), 319-324.

[30] T.R. Jow, S.A. Delp, J.L. Allen, J.-P. Jones, M.C. Smart, J. Electrochem. Soc., 2018, 165(2), A361.

[31] Y. Tang, Y. Zhang, W. Li, B. Ma, X. Chen, Chem. Soc. Rev. 2015, 44(17), 5926-5940.

[32] H. Gao, Q. Wu, Y. Hu, J.P. Zheng, K. Amine, Z. Chen, J. Phys. Chem. Lett. 2018, 9(17), 5100-5104.

[33] P. Arora, Z. Zhang, Chem. Rev 2004, 104, 4419-4462.

[34] R. Pan, Z. Wang, R. Sun, J. Lindh, K. Edström, M. Strømme, L. Nyholm, Cellulose 2017, 24(7), 2903-2911.

[35] S.J. Kim, M.C. Kim, D.H. Kwak, D.M. Kim, G.H. Lee, H.S. Choe, K.W. Park, J. Power Sources,. 2016, 304, 119-127.

[36] S. Kalluri, M. Yoon, M. Jo, S. Park, S. Myeong, J. Kim,
S.X. Dou, Z. Guo, J. Cho, Adv. Energy Mater, 2017, 7(1), 1601507

[37] J. Qian, L. Liu, J. Yang, S. Li, X. Wang, H.L. Zhuang, Y. Lu, Nat. Commun., 2018, 9(1), 1-11.

[38] W. Lee, D. Lee, Y. Kim, W. Choi, W.-S. Yoon, J. Mater. Chem. A 2020, 8(20), 10206-10216.

[39] K.X. Wang, X.H. Li, J.S. Chen, Adv. Mater, 2015, 27(3), 527-545.

[40] C. Jiang, M. Wei, Z. Qi, T. Kudo, I. Honma, H. Zhou, J. Power Sources 2007, 166(1), 239-243.

[41] H. Liu, A. Banerjee, B. Ziv, K.J. Harris, N.P.W. Pieczonka, S. Luski, G.A. Botton, G.R. Goward, D. Aurbach, I.C. Halalay, ACS Appl. Energy Mater. 2018, 1(5), 1878-1882.

[42] M. Ling, J. Qiu, S. Li, C. Yan, M.J. Kiefel, G. Liu, S. Zhang, Nano Lett. 2015, 15(7), 4440-4447.

[43] J. Wang, N. Yang, H. Tang, Z. Dong, Q. Jin, M. Yang,D. Kisailus, H. Zhao, Z. Tang, D. Wang, Angew. Chemie., 2013, 52(25), 6417-6420.

[44] J.M. Feckl, K. Fominykh, M. Dblinger, D. FattakhovaRohlfing, T. Bein, Angew. Chemie., 2012, 51(30),7459-7463.

[45] Z.S. Wu, W. Ren, L. Xu, F. Li, H.M. Cheng, ACS Nano 2011, 5(7), 5463-5471.

[46] W. Lee, S. Muhammad, T. Kim, H. Kim, E. Lee, M. Jeong, S. Son, J.H. Ryou, W.S. Yoon, Adv. Energy Mater. 2018, 8(4), 1701788.

[47] A. Magasinski, P. Dixon, B. Hertzberg, A. Kvit, J. Ayala, G. Yushin, Nat. Mater,. 2010, 9(4), 353-358. 